\title{
Determination of QCD phase diagram from the imaginary chemical potential region
}

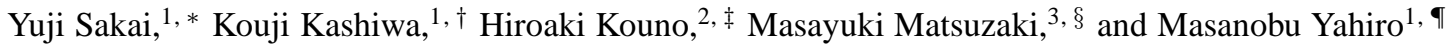 \\ ${ }^{1}$ Department of Physics, Graduate School of Sciences, Kyushu University, Fukuoka 812-8581, Japan \\ ${ }^{2}$ Department of Physics, Saga University, Saga 840-8502, Japan \\ ${ }^{3}$ Department of Physics, Fukuoka University of Education, Munakata, Fukuoka 811-4192, Japan
}

(Dated: October 28, 2018)

\begin{abstract}
We test the reliability of the the Polyakov-loop extended Nambu-Jona-Lasinio (PNJL) model, comparing the model result with the lattice data at nonzero imaginary chemical potential. The PNJL model with the vector-type four-quark and scalar-type eight-quark interactions reproduces the lattice data on the pseudocritical temperatures of the deconfinement and chiral phase transitions. The QCD phase diagram in the real chemical potential region is predicted by the PNJL model. The critical endpoint survives, even if the vector-type four-quark interaction is taken into account.
\end{abstract}

PACS numbers: 11.30.Rd, 12.40.-y

\section{INTRODUCTION}

Quantum Chromodynamics (QCD) is a remarkable theory. It is renormalizable and essentially parameter free. QCD accounts for the rich phenomenology of hadronic and nuclear physics. Thermodynamics of QCD is also well defined. Nevertheless, it is not well known because of its nonperturbative nature. In particular, QCD phase diagram is essential for understanding not only natural phenomena such as compact stars and the early universe but also laboratory experiments such as relativistic heavy-ion collisions.

Unfortunately, quantitative calculations of the phase diagram from first-principle lattice QCD (LQCD) have the well known sign problem when the chemical potential $(\mu)$ is real; for example, see Ref. [1] and references therein. So far, several approaches have been proposed to circumvent the difficulty; for example, the reweighting method [2], the Taylor expansion method [3] and the analytic continuation to real chemical potential $\left(\mu_{\mathrm{R}}\right)$ from imaginary chemical potential $\left(\mu_{\mathrm{I}}\right)$ [4, 5, 6, 7, 8]. However, those are still far from perfection.

As an approach complementary to first-principle lattice QCD, we can consider effective models such as the NambuJona-Lasinio (NJL) model [9, 10, 11, 12, 13, 14, 15, 16, 17] and the Polyakov-loop extended Nambu-Jona-Lasinio (PNJL) model [18, 19, 20, 21, 22, 23, 24, 25, 26, 27, 28, 29, 30, 31, 32, 33, 34, 35]. The NJL model describes the chiral symmetry breaking, but not the confinement mechanism. The PNJL model is designed [20] to make it possible to treat the Polyakov loop as well as the chiral symmetry breaking.

In the NJL-type models, the input parameters are determined at $\mu=0$ and $T \geq 0$, where $T$ is temperature. It is then highly nontrivial whether the models predict properly dynamics of QCD at finite $\mu_{\mathrm{R}}$. This should be tested from QCD.

\footnotetext{
*sakai@phys.kyushu-u.ac.jp

†kashiwa@phys.kyushu-u.ac.jp

抽ounoh@cc.saga-u.ac.jp

$\S$ matsuza@fukuoka-edu.ac.jp

"yahiro@phys.kyushu-u.ac.jp
}

Fortunately, this is possible in the $\mu_{\mathrm{I}}$ region, since lattice QCD has no sign problem there. The canonical partition function $Z_{\mathrm{C}}(n)$ with real quark number $n$ is the Fourier transform of the grand-canonical one $Z_{\mathrm{GC}}(\theta)$ with $\theta=\mu_{\mathrm{I}} / T$ [36]:

$$
Z_{\mathrm{C}}(n)=\frac{1}{2 \pi} \int_{-\pi}^{\pi} d \theta e^{-i n \theta} Z_{\mathrm{GC}}(\theta) .
$$

Thus, the thermodynamic potential of QCD, $\Omega_{\mathrm{QCD}}(\theta)=$ $-T \ln \left(Z_{\mathrm{GC}}(\theta)\right)$, at finite $\theta$ includes all dynamics at real $n$ and hence at finite $\mu_{\mathrm{R}}$. Therefore, the reliability of effective models at finite $\mu_{\mathrm{R}}$ can be tested in the $\mu_{\mathrm{I}}$ region.

Roberge and Weiss found [36] that QCD has a periodicity $\Omega_{\mathrm{QCD}}(\theta)=\Omega_{\mathrm{QCD}}(\theta+2 \pi k / 3)$, showing that $\Omega_{\mathrm{QCD}}(\theta+$ $2 \pi k / 3)$ is transformed into $\Omega_{\mathrm{QCD}}(\theta)$ by the $\mathbb{Z}_{3}$ transformation with integer $k$. This means that QCD is invariant under a combination of the $\mathbb{Z}_{3}$ transformation and a parameter transformation $\theta \rightarrow \theta+2 k \pi / 3$ [34, 35],

$$
\begin{aligned}
q & \rightarrow U q, \quad A_{\nu} \rightarrow U A_{\nu} U^{-1}-i / g\left(\partial_{\nu} U\right) U^{-1}, \\
\theta & \rightarrow \theta+2 \pi k / 3
\end{aligned}
$$

where $U(x, \tau)$ are elements of SU(3) with $U(x, \beta=1 / T)=$ $\exp (-2 i \pi k / 3) U(x, 0)$ and $q$ is the quark field. We call this combination the extended $\mathbb{Z}_{3}$ transformation. Thus, $\Omega_{\mathrm{QCD}}(\theta)$ has the extended $\mathbb{Z}_{3}$ symmetry, and hence quantities invariant under the extended $\mathbb{Z}_{3}$ transformation have the RW periodicity [34, 35]. At the present stage, the PNJL model is only a realistic effective model that possesses both the extended $\mathbb{Z}_{3}$ symmetry and chiral symmetry [34, 35]. This property makes it possible to compare PNJL with lattice QCD quantitatively in the $\mu_{\mathrm{I}}$ region. If the PNJL model succeeds in reproducing the lattice data, we may think that the PNJL model will predict, with high reliability, the QCD phase structure in the $\mu_{\mathrm{R}}$ region.

The extended $\mathbb{Z}_{3}$ symmetry in QCD is a remnant of the $\mathbb{Z}_{3}$ symmetry, namely the confinement mechanism, in the pure gauge system. The extended $\mathbb{Z}_{3}$ symmetry appears as the RW periodicity in the $\mu_{\mathrm{I}}$ region and implicitly affects dynamics in the $\mu_{\mathrm{R}}$ region. Actually, the mechanism largely shifts the critical endpoint [10] toward higher $T$ and lower $\mu$ than the NJL model predicts [21, 25, 31]. In contrast, the vector-type fourquark interaction $G_{\mathrm{V}}\left(\bar{q} \gamma_{\mu} q\right)^{2}$ largely moves the critical endpoint in the opposite direction [14, 16, 21, 31], if it is newly 
added to the NJL and PNJL models. Thus, it is essential to determine the strength of the coupling $G_{\mathrm{v}}$ of the vector-type interaction, although the interaction is often ignored in the NJL and PNJL calculations.

In the relativistic meson-nucleon theory [37], the repulsive force mediated by vector mesons is essential to account for the saturation property of nuclear matter. Using the auxiliary field method, one can convert quark-quark interactions to mesonquark interactions; for example, see Refs. [17, 38, 39] and references therein. In the hadron phase, quarks have a large effective mass as a result of spontaneous chiral symmetry breaking, and then nucleons can be considered to be formed by such three heavy quarks, i.e. three constituent quarks. It is then natural to think that there exists the correspondence between the meson-nucleon interactions and the quark-quark interactions. In this sense, it is very likely that the vector-type four-quark interaction is not negligible and even significant in particular at a finite quark-density region corresponding to the nuclear saturation density. In the previous work [35], we have proposed that the strength of $G_{\mathrm{v}}$ can be determined from lattice data on the chiral phase transition in the $\mu_{\text {I }}$ region.

In this paper, we consider two-flavor QCD and show the reliability of the PNJL model, quantitatively comparing the model result with lattice data in the $\mu_{\mathrm{I}}$ region. The model parameters except $G_{\mathrm{V}}$ are fixed by the measured pion mass and decay constant at $\mu=T=0$ and lattice data [40, 41, 42] at $T>0$ and $\mu=0$. The PNJL calculation with no vector-type interaction well reproduces lattice data [4, 8] on the pseudocritical temperature $T_{\mathrm{c}}(\Phi)$ of the deconfinement phase transition, but not on the pseudocritical temperature $T_{\mathrm{c}}(\sigma)$ of the chiral phase transition near $\theta=\pi / 3$. The strength of $G_{\mathrm{v}}$ is fitted so as to reproduce the latter data. The primary result of the lattice simulations is that $T_{\mathrm{c}}(\Phi)$ coincides with $T_{\mathrm{c}}(\sigma)$, within numerical errors, in the entire region of $\theta$ [4, 8]. The PNJL model with the vector-type interaction can reproduce this property. Finally, we quantitatively predict the phase diagram in the $\mu_{\mathrm{R}}$ region by using the PNJL model with the parameter set justified in the $\mu_{\mathrm{I}}$ region. This sort of model predictions are quite important before doing heavy lattice calculations with large lattice size in the $\mu_{\mathrm{I}}$ region.

In section II, the PNJL model is explained simply. In section III, we test the PNJL model in the $\mu_{\mathrm{I}}$ region and determine the strength of $G_{\mathrm{v}}$. Finally, we predict the phase diagram in the $\mu_{\mathrm{R}}$ region. Section IV is devoted to summary.

\section{PNJL MODEL}

The two-flavor PNJL Lagrangian is

$$
\begin{aligned}
\mathcal{L}= & \bar{q}\left(i \gamma_{\nu} D^{\nu}-m_{0}\right) q \\
& +G_{\mathrm{S}}\left[(\bar{q} q)^{2}+\left(\bar{q} i \gamma_{5} \vec{\tau} q\right)^{2}\right]-\mathcal{U}\left(\Phi[A], \Phi[A]^{*}, T\right),
\end{aligned}
$$

where $q$ denotes the two-flavor quark field, $m_{0}$ does the current quark mass, and $D^{\nu}=\partial^{\nu}+i A^{\nu}-i \mu \delta_{0}^{\nu}$. The field $A^{\nu}$ is defined as $A^{\nu}=\delta_{0}^{\nu} g A_{a}^{0} \frac{\lambda^{a}}{2}$ with the gauge field $A_{a}^{\nu}$, the GellMann matrix $\lambda_{a}$ and the gauge coupling $g$. In the NJL sector, $G_{\mathrm{S}}$ denotes the coupling constant of the scalar-type four-quark interaction. Later, we will add the vector-type four-quark interaction [10, 14, 16, 35] and the scalar-type eight-quark interaction [15, 16, 34] to the PNJL Lagrangian. The Polyakov potential $\mathcal{U}$, defined in $(8)$, is a function of the Polyakov loop $\Phi$ and its Hermitian conjugate $\Phi^{*}$,

$$
\Phi=\frac{1}{N_{\mathrm{c}}} \operatorname{Tr} L, \quad \Phi^{*}=\frac{1}{N_{\mathrm{c}}} \operatorname{Tr} L^{\dagger},
$$

with

$$
L(\mathbf{x})=\mathcal{P} \exp \left[i \int_{0}^{\beta} d \tau A_{4}(\mathbf{x}, \tau)\right]
$$

where $\mathcal{P}$ is the path ordering and $A_{4}=i A_{0}$. In the chiral limit $\left(m_{0}=0\right)$, the Lagrangian density has the exact $S U\left(N_{f}\right)_{\mathrm{L}} \times$ $S U\left(N_{f}\right)_{\mathrm{R}} \times U(1)_{\mathrm{v}} \times S U(3)_{\mathrm{c}}$ symmetry.

The temporal component of the gauge field is diagonal in the flavor space, because the color and the flavor space are completely separated out in the present case. In the Polyakov gauge, $L$ can be written in a diagonal form in the color space [20]:

$$
L=e^{i \beta\left(\phi_{3} \lambda_{3}+\phi_{8} \lambda_{8}\right)}=\operatorname{diag}\left(e^{i \beta \phi_{a}}, e^{i \beta \phi_{b}}, e^{i \beta \phi_{c}}\right),
$$

where $\phi_{a}=\phi_{3}+\phi_{8} / \sqrt{3}, \phi_{b}=-\phi_{3}+\phi_{8} / \sqrt{3}$ and $\phi_{c}=$ $-\left(\phi_{a}+\phi_{b}\right)=-2 \phi_{8} / \sqrt{3}$. The Polyakov loop $\Phi$ is an exact order parameter of the spontaneous $\mathbb{Z}_{3}$ symmetry breaking in the pure gauge theory. Although the $\mathbb{Z}_{3}$ symmetry is not an exact one in the system with dynamical quarks, it still seems to be a good indicator of the deconfinement phase transition. Therefore, we use $\Phi$ to define the deconfinement phase transition.

Making the mean field approximation and performing the path integral over quark field, one can obtain the thermodynamic potential $\Omega$ (per volume),

$$
\begin{aligned}
\Omega= & -2 N_{f} \int \frac{d^{3} \mathrm{p}}{(2 \pi)^{3}}[3 E(\mathrm{p}) \\
& +\frac{1}{\beta} \ln \left[1+3\left(\Phi+\Phi^{*} e^{-\beta E^{-}(\mathbf{p})}\right) e^{-\beta E^{-}(\mathbf{p})}+e^{-3 \beta E^{-}(\mathbf{p})}\right] \\
& \left.+\frac{1}{\beta} \ln \left[1+3\left(\Phi^{*}+\Phi e^{-\beta E^{+}(\mathbf{p})}\right) e^{-\beta E^{+}(\mathbf{p})}+e^{-3 \beta E^{+}(\mathbf{p})}\right]\right] \\
& +U_{\mathrm{M}}+\mathcal{U} .
\end{aligned}
$$

where, $\sigma=\langle\bar{q} q\rangle, \Sigma_{\mathrm{s}}=-2 G_{\mathrm{s}} \sigma, M=m_{0}+\Sigma_{\mathrm{s}}, U_{\mathrm{M}}=G_{\mathrm{s}} \sigma^{2}$, $E(\mathrm{p})=\sqrt{\mathrm{p}^{2}+M^{2}}$ and $E^{ \pm}(\mathrm{p})=E(\mathrm{p}) \pm \mu=E(\mathrm{p}) \pm i \theta / \beta$. In (7), only the first term of the right-hand side diverges. It is then regularized by the three-dimensional momentum cutoff $\Lambda$ [20, 24]. We use $\mathcal{U}$ of Ref. [25] that is fitted to a lattice QCD simulation in the pure gauge theory at finite $T[43,44]$ :

$$
\begin{aligned}
& \mathcal{U}=T^{4}\left[-\frac{a(T)}{2} \Phi^{*} \Phi\right. \\
& \left.\quad+b(T) \ln \left(1-6 \Phi \Phi^{*}+4\left(\Phi^{3}+\Phi^{* 3}\right)-3\left(\Phi \Phi^{*}\right)^{2}\right)\right] \\
& a(T)=a_{0}+a_{1}\left(\frac{T_{0}}{T}\right)+a_{2}\left(\frac{T_{0}}{T}\right)^{2}, \quad b(T)=b_{3}\left(\frac{T_{0}}{T}\right)^{3}
\end{aligned}
$$


where parameters are summarized in Table I. The Polyakov potential yields a first-order deconfinement phase transition at $T=T_{0}$ in the pure gauge theory. The original value of $T_{0}$ is $270 \mathrm{MeV}$ evaluated by the pure gauge lattice QCD calculation. However, the PNJL model with this value of $T_{0}$ yields somewhat larger value of the transition temperature at zero chemical potential than the full LQCD simulation [40, 41, 42] predicts. Therefore, we rescale $T_{0}$ to $212 \mathrm{MeV}$; the detail will be shown in subsection

\begin{tabular}{cccc}
\hline$a_{0}$ & $a_{1}$ & $a_{2}$ & $b_{3}$ \\
\hline 3.51 & -2.47 & 15.2 & -1.75 \\
\hline
\end{tabular}

TABLE I: Summary of the parameter set in the Polyakov sector used in Ref. [25]. All parameters are dimensionless.

The variables $X=\Phi, \Phi^{*}$ and $\sigma$ satisfy the stationary conditions,

$$
\partial \Omega / \partial X=0 .
$$

The solutions of the stationary conditions do not give the global minimum $\Omega$ necessarily. There is a possibility that they yield a local minimum or even a maximum. We then have checked that the solutions yield the global minimum when the solutions $X(\theta)$ are inserted into (7).

The thermodynamic potential $\Omega$ of Eq. (7) is not invariant under the $\mathbb{Z}_{3}$ transformation,

$$
\Phi(\theta) \rightarrow \Phi(\theta) e^{-i 2 \pi k / 3}, \quad \Phi(\theta)^{*} \rightarrow \Phi(\theta)^{*} e^{i 2 \pi k / 3},
$$

although $\mathcal{U}$ of $(8)$ is invariant. Instead of the $\mathbb{Z}_{3}$ symmetry, however, $\Omega$ is invariant under the extended $\mathbb{Z}_{3}$ transformation,

$$
\begin{aligned}
& e^{ \pm i \theta} \rightarrow e^{ \pm i \theta} e^{ \pm i \frac{2 \pi k}{3}}, \quad \Phi(\theta) \rightarrow \Phi(\theta) e^{-i \frac{2 \pi k}{3}}, \\
& \Phi(\theta)^{*} \rightarrow \Phi(\theta)^{*} e^{i \frac{2 \pi k}{3}} .
\end{aligned}
$$

This is easily understood as follows. It is convenient to introduce the modified Polyakov loop $\Psi \equiv e^{i \theta} \Phi$ and $\Psi^{*} \equiv e^{-i \theta} \Phi^{*}$ invariant under the transformation (12). The extended $\mathbb{Z}_{3}$ transformation is then rewritten into

$$
\begin{aligned}
& e^{ \pm i \theta} \rightarrow e^{ \pm i \theta} e^{ \pm i \frac{2 \pi k}{3}}, \quad \Psi(\theta) \rightarrow \Psi(\theta), \\
& \Psi(\theta)^{*} \rightarrow \Psi(\theta)^{*},
\end{aligned}
$$

and $\Omega$ is also into

$$
\begin{aligned}
\Omega= & -2 N_{f} \int \frac{d^{3} \mathrm{p}}{(2 \pi)^{3}}\left[3 E(\mathrm{p})+\frac{1}{\beta} \ln \left[1+3 \Psi e^{-\beta E(\mathbf{p})}\right.\right. \\
& \left.+3 \Psi^{*} e^{-2 \beta E(\mathbf{p})} e^{\beta \mu_{\mathrm{B}}}+e^{-3 \beta E(\mathbf{p})} e^{\beta \mu_{\mathrm{B}}}\right] \\
& +\frac{1}{\beta} \ln \left[1+3 \Psi^{*} e^{-\beta E(\mathbf{p})}+3 \Psi e^{-2 \beta E(\mathbf{p})} e^{-\beta \mu_{\mathrm{B}}}\right. \\
& \left.\left.+e^{-3 \beta E(\mathbf{p})} e^{-\beta \mu_{\mathrm{B}}}\right]\right]+U_{\mathrm{M}}+\mathcal{U}
\end{aligned}
$$

where $\beta \mu_{\mathrm{B}}=3 \beta \mu=3 i \theta$. Obviously, $\Omega$ is invariant under the extended $\mathbb{Z}_{3}$ transformation (13), since it is a function of only extended $\mathbb{Z}_{3}$ invariant quantities, $e^{3 i \theta}$ and $\tilde{X}\left(=\Psi, \Psi^{*}, \sigma\right)$.
The explicit $\theta$ dependence appears only through the factor $e^{3 i \theta}$ in (14). Hence, the stationary conditions (10) show that $\tilde{X}=\tilde{X}\left(e^{3 i \theta}\right)$. Inserting the solutions back to (14), one can see that $\Omega=\Omega\left(e^{3 i \theta}\right)$. Thus, $\tilde{X}$ and $\Omega$ have the RW periodicity,

$$
\tilde{X}\left(\theta+\frac{2 \pi k}{3}\right)=\tilde{X}(\theta), \quad \text { and } \quad \Omega\left(\theta+\frac{2 \pi k}{3}\right)=\Omega(\theta),
$$

while the Polyakov loop $\Phi$ and its Hermitian conjugate $\Phi^{*}$ have the properties

$$
\begin{aligned}
\Phi\left(\theta+\frac{2 \pi k}{3}\right) & =e^{-i 2 \pi k / 3} \Phi(\theta), \\
\Phi\left(\theta+\frac{2 \pi k}{3}\right)^{*} & =e^{i 2 \pi k / 3} \Phi(\theta)^{*} .
\end{aligned}
$$

\section{NUMERICAL RESULTS}

\section{A. Thermal system with no chemical potential}

First, we consider the thermal system with no chemical potential to determine the parameters, $m_{0}, G_{\mathrm{s}}, \Lambda$ and $T_{0}$ of the PNJL model. In the lattice calculations [40, 41, 42], the pseudocritical temperature $T_{\mathrm{c}}(\sigma)$ of the crossover chiral phase transition coincides with that $T_{\mathrm{C}}(\Phi)$ of the crossover deconfinement one within $10 \%$ error: $T_{\mathrm{c}}(\sigma) \approx T_{\mathrm{c}}(\Phi) \approx$ $173 \pm 8 \mathrm{MeV}$ [41].

The parameter set, $\Lambda=631.5 \mathrm{MeV}, G_{\mathrm{s}}=5.498\left[\mathrm{GeV}^{-2}\right]$ and $m_{0}=5.5 \mathrm{MeV}$, can reproduce the pion decay constant $f_{\pi}=93.3 \mathrm{MeV}$ and the pion mass $M_{\pi}=138 \mathrm{MeV}$ at $T=\mu=0$ [16], and keeps a good reproduction also at finite $T$ [25]. We then adopt these values for $\Lambda, G_{\mathrm{S}}$ and $m_{0}$. We adjust $T_{0}$ so that the PNJL calculation can reproduce the lattice result $T_{\mathrm{c}}(\Phi)=173 \mathrm{MeV}$; the value is $T_{0}=212 \mathrm{MeV}$. The parameter set thus determined is shown as set A in Table

\begin{tabular}{cccc}
\hline set & $G_{\mathrm{s}}$ & $G_{\mathrm{s} 8}$ & $G_{\mathrm{v}}$ \\
\hline A & $5.498 \mathrm{GeV}^{-2}$ & 0 & 0 \\
\hline B & $4.673 \mathrm{GeV}^{-2}$ & $452.12 \mathrm{GeV}^{-8}$ & 0 \\
\hline C & $4.673 \mathrm{GeV}^{-2}$ & $452.12 \mathrm{GeV}^{-8}$ & $4.673 \mathrm{GeV}^{-2}$ \\
\hline
\end{tabular}

TABLE II: Summary of the parameter sets in the PNJL calculations. The parameters $\Lambda, m_{0}$ and $T_{0}$ are common among the three sets; $\Lambda=631.5 \mathrm{MeV}, m_{0}=5.5 \mathrm{MeV}$ and $T_{0}=212 \mathrm{MeV}$.

Figure 11 shows the chiral condensate $\sigma$ normalized by $\sigma_{0}=\left.\sigma\right|_{T=0, \mu=0}$ and the absolute value of the Polyakov loop $\Phi$ as a function of $T / T_{\mathrm{c}}$. In this paper $T_{\mathrm{c}}$ is always taken to be $173 \mathrm{MeV}$. The green curves represent the PNJL results of parameter set $\mathrm{A}$, where $\sigma_{0}=-0.0302\left[\mathrm{GeV}^{3}\right]$ in this case. Lattice QCD data [40, 41, 42] are also plotted by cross symbols with $10 \%$ error bar; $\sigma$ and $|\Phi|$ measured as a function of $T / T_{\mathrm{c}}$ in Refs. [40, 41, 42] have only small errors, but we have added $10 \%$ error that the lattice calculation [41] has in determining $T_{\mathrm{c}}$. For $|\Phi|$ the PNJL result (green solid curve) reasonably agrees with the lattice one $(\times)$. For $\sigma$, however, 
the PNJL result (green dashed curve) considerably overshoots the lattice data $(+)$.

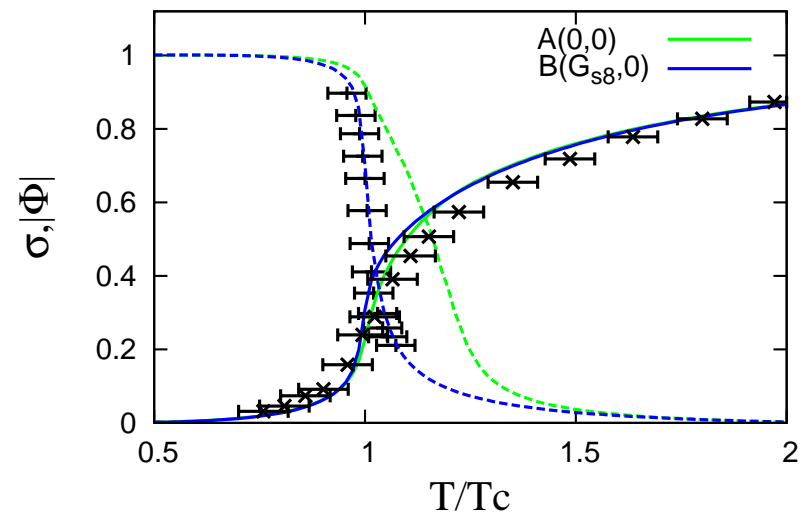

Fig. 1: Chiral condensate $\sigma$ normalized by $\sigma(T=0, \mu=0)$ and the absolute value of the Polyakov loop $\Phi$. The blue (green) curve represents the PNJL result of parameter set B (A) with (without) the scalar-type eight-quark interaction; $\sigma(|\Phi|)$ is denoted by the dashed (solid) curve. Lattice data ( + ) on $\sigma$ are taken from Ref. [40] and those $(\times)$ on $|\Phi|$ are from Ref. [42]. The lattice data are plotted with $10 \%$ error bar, since lattice calculations have $10 \%$ error in determining $T_{\mathrm{c}}$ [41].

Figure 2 represents results of the PNJL calculations for chiral and Polyakov-loop susceptibilities, $\chi_{\sigma}$ and $\chi_{\Phi}$ [21]. Peak positions of $\chi_{\sigma}$ and $\chi_{\Phi}$ show $T_{\mathrm{c}}(\sigma)$ and $T_{\mathrm{c}}(\Phi)$, respectively. The PNJL results (green curves) of parameter set A give $T_{\mathrm{c}}(\sigma) / T_{\mathrm{c}}=1.25$ and $T_{\mathrm{c}}(\Phi) / T_{\mathrm{c}}=1$, while the lattice simulations yield $T_{\mathrm{c}}(\sigma) / T_{\mathrm{c}}=1 \pm 0.05$ and $T_{\mathrm{c}}(\Phi) / T_{\mathrm{c}}=$ $1 \pm 0.05$. The PNJL results are consistent with the lattice ones for $T_{\mathrm{c}}(\Phi)$, but not for $T_{\mathrm{c}}(\sigma)$.

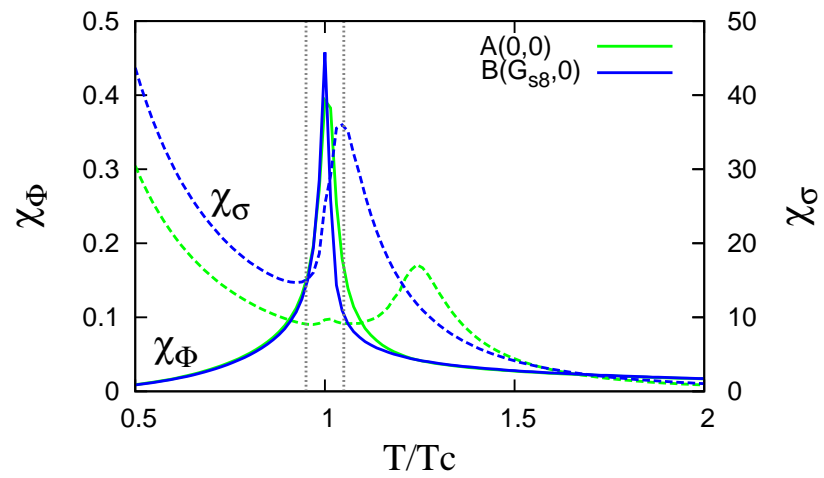

Fig. 2: $T$ dependence of chiral and Polyakov-loop susceptibilities, $\chi_{\sigma}$ (right scale) and $\chi_{\Phi}$ (left scale). The blue (green) curve represents the PNJL result of parameter set B (A) with (without) the scalar-type eight-quark interaction; $\chi_{\sigma}\left(\chi_{\Phi}\right)$ is denoted by the dashed (solid) curve. The region between two vertical gray lines $T=(1 \pm 0.05) T_{\mathrm{c}}$ is the prediction of lattice calculations [41].

Now we introduce the scalar-type eight-quark interaction [16],

$$
G_{\mathrm{s} 8}\left[(\bar{q} q)^{2}+\left(\bar{q} i \gamma_{5} \vec{\tau} q\right)^{2}\right]^{2}
$$

since the difference $T_{\mathrm{c}}(\sigma)-T_{\mathrm{c}}(\Phi)$ is reduced by the interaction [34].

Since $f_{\pi}$ and $M_{\pi}$ calculated with PNJL depend on the strength of $G_{\mathrm{s} 8}$, for each value of $G_{\mathrm{s} 8}$ the strength of $G_{\mathrm{S}}$ is re-adjusted so as to reproduce the measured values $f_{\pi}=$ $93.3 \mathrm{MeV}$ and $M_{\pi}=138 \mathrm{MeV}$. As $G_{\mathrm{s} 8}$ increases from zero, $T_{\mathrm{c}}(\sigma)$ calculated with PNJL decreases toward $T_{\mathrm{c}}=173 \mathrm{MeV}$. When $G_{\mathrm{s} 8}=452.12 \mathrm{GeV}^{-8}$, the ratio $T_{\mathrm{c}}(\sigma) / T_{\mathrm{c}}$ becomes 1.05 and hence consistent with the corresponding lattice result within $10 \%$ error. We adopt this strength. This parameter set is shown as set B in Table [I] As shown in Fig. 1, the PNJL results (blue curves) of parameter set B well reproduce the lattice results for both the chiral condensate and the Polyakov loop.

\section{B. Thermal system with imaginary chemical potential}

In this subsection, we consider the thermal system with finite imaginary chemical potential and compare the PNJL result with the lattice data [4]([8]) in which the lattice size is $8^{3} \times 4$ and the two-flavor $\mathrm{KS}$ (Wilson) fermion is considered.

First, we analyze the deconfinement phase transition. Since the eight-quark interaction hardly changes the Polyakov loop, we do the PNJL calculation with parameter set A. Figure 3 presents $T$ dependence of the Polyakov-loop susceptibility $\chi_{\Phi}$ in three cases of $\theta=0,0.56$ and 0.96; each case is distinguished by using different colors. For each $\theta$, the PNJL result (solid curve) reproduces the corresponding lattice result (crosses) in its peak position. Thus, the PNJL results are consistent with the lattice ones for the pseudocritical temperature of the crossover deconfinement phase transition.

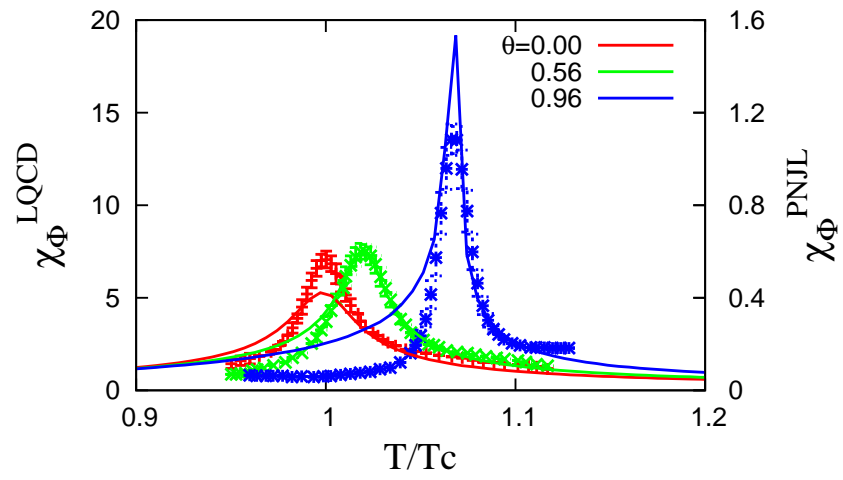

Fig. 3: $T$ dependence of the Polyakov-loop susceptibilities in three cases of $\theta=0,0.56$ and 0.96 ; each case is distinguished by using different colors. The solid curves represent the PNJL results of set A (right scale). Lattice data shown by crosses (left scale) are taken from Ref. [8].

Figure 4 presents the phase diagram of the deconfinement phase transition in the $\theta-T$ plane, where $\theta$ is divided by $\pi / 3$ and $T$ is normalized by $T_{\mathrm{c}}=173 \mathrm{MeV}$. Lattice data [8] measured as a function of $T / T_{\mathrm{c}}$ have only small errors, as shown by thick error bars in Fig. 4 . This is an error bar in the case 
that lattice calculations have no error in $T_{\mathrm{c}}$. However, the lattice calculation [41] has about $10 \%$ error in determining $T_{\mathrm{c}}$, as mentioned in subsection IIIA. This $10 \%$ error should be added to the original small error; this $10 \%$ error will be shown later in Fig. 6. The PNJL result (solid curve) of set A agrees with the lattice one (crosses) within the error bars. The phase diagram has a periodicity of $2 \pi / 3$ in $\theta$. This is called the Roberge and Weiss (RW) periodicity [36]. The phase diagram is also $\theta$ even, because so is $\chi_{\Phi}$. On the dot-dashed line going up from an endpoint $\left(\theta_{\mathrm{RW}}, T_{\mathrm{RW}}\right)=\left(\pi / 3,1.09 T_{\mathrm{c}}\right)$, the quark number density $n$ and the phase $\phi$ of the Polyakov loop are discontinuous in the PNJL calculations [34, 35]. This is called the RW phase transition line. The lattice data [4, 8] on $\phi$ are also discontinuous on the line, as shown later in Fig 7 Thus, the PNJL result is consistent with the lattice results [4, 8] also for the location of the RW phase transition line.

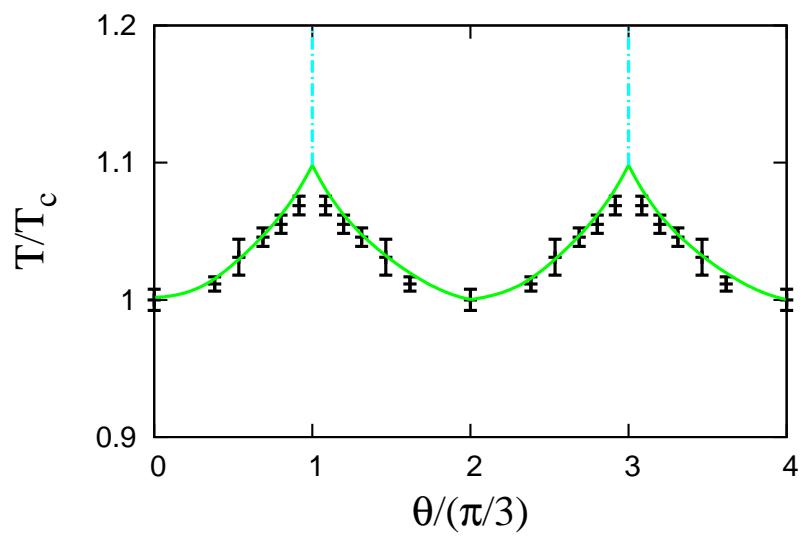

Fig. 4: Phase diagram on the $\theta-T$ plane. The solid curve represents the deconfinement phase transition, while the dot-dashed line does the RW phase transition predicted by the PNJL calculation with set A. Lattice data are taken from Ref. [8].

The lattice simulations [4, 8] point out that $T_{\mathrm{c}}(\sigma)$ agrees with $T_{\mathrm{c}}(\Phi)$ within numerical errors in the entire region $0 \leq$ $\theta \leq 2 \pi / 3$. We then take the case of $\theta=\pi / 3$ to consider this point. It is predicted by the lattice simulations that $T_{\mathrm{c}}(\sigma)$ and $T_{\mathrm{c}}(\Phi)$ are located in the region between two vertical gray lines of Fig. 5. Panel (a) shows $\sigma$ and $|\Phi|$ as a function $T / T_{\mathrm{c}}$ and panel (b) does $\chi_{\sigma}$ and $\chi_{\Phi}$ as a function $T / T_{\mathrm{c}}$. The green (blue) curves represent results of the PNJL calculations with set A (B). The eight-quark interaction hardly shifts the peak position of $\chi_{\Phi}$, i.e. $T_{\mathrm{c}}(\Phi)$, from the value $1.09 T_{\mathrm{c}}$. The peak position is consistent with the lattice result shown by the region between two vertical gray lines. In contrast, the eight-quark interaction largely shifts the peak position of $\chi_{\sigma}$, i.e. $T_{\mathrm{c}}(\sigma)$, from $1.53 T_{\mathrm{c}}$ to $1.24 T_{\mathrm{c}}$, but the shifted value still deviates from $T_{\mathrm{c}}(\Phi)=$ $(1.1 \pm 0.05) T_{\mathrm{c}}$, that is, the lattice data near $\theta=\pi / 3$ [4, 8] shown by the region between two vertical gray lines.

In order to solve this problem, we introduce the vector-type four-quark interaction

$$
-G_{\mathrm{v}}\left(\bar{q} \gamma_{\mu} q\right)^{2}
$$

and add it to the PNJL Lagrangian $\mathcal{L}$; see Ref. [35] for the detail of this formulation. As mentioned in Ref. [35], the phase structure in the real chemical potential region is quite sensitive to the strength of the coupling $G_{\mathrm{v}}$. It is then important to determine the strength, but it has not been done yet. Since the vector-type interaction does not change the pion mass and the pion decay constant at $T=\mu=0$ and the chiral condensate and the Polyakov loop at $T \geq 0$ and $\mu=0$, we can simply add the interaction to set B. As $G_{\mathrm{v}}$ increases from zero, $T_{\mathrm{c}}(\sigma)$ goes down toward $T_{\mathrm{c}}(\Phi)$, while $T_{\mathrm{c}}(\Phi)$ moves little. When $G_{\mathrm{v}}=4.673 \mathrm{GeV}^{-2}, T_{\mathrm{c}}(\sigma)$ gets into the region between the vertical gray lines. We adopt this strength of $G_{\mathrm{v}}$. This set is shown as set $\mathrm{C}$ in Table
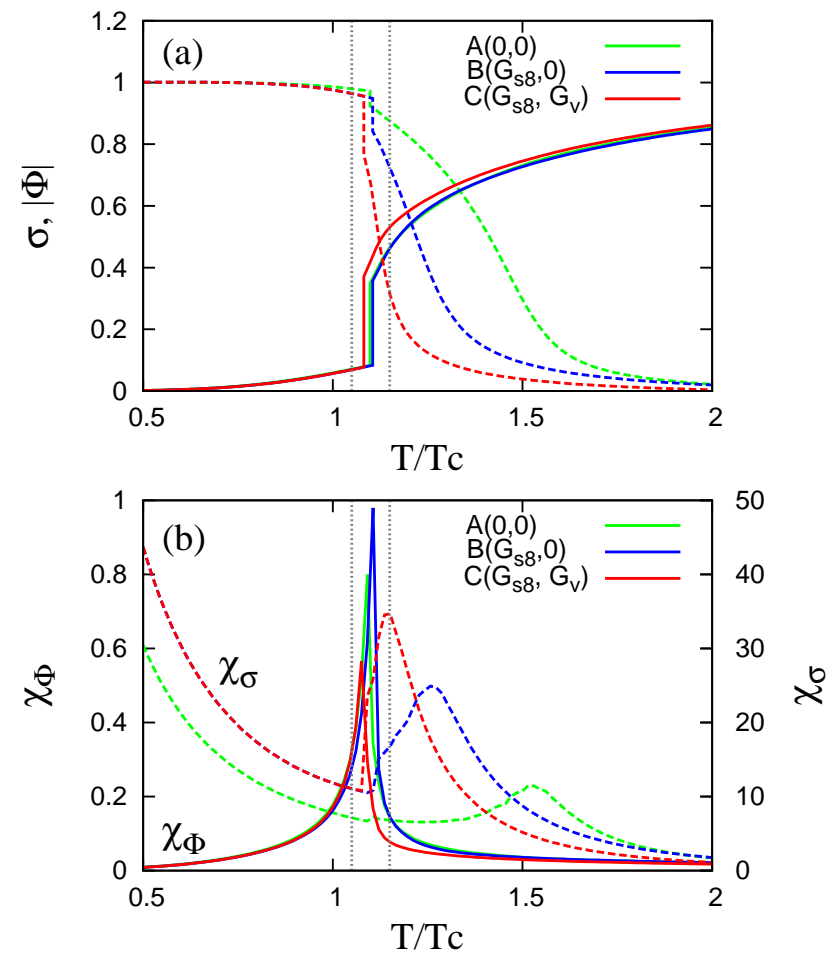

Fig. 5: $T$ dependence of (a) the normalized chiral condensate and the absolute value of the Polyakov loop and (b) the susceptibilities $\chi_{\sigma}$ (right scale) and $\chi_{\Phi}$ (left scale) at $\theta=\pi / 3$. In panel (a), $\sigma$ $(|\Phi|)$ is denoted by the dashed (solid) curve. In panel (b), $\chi_{\sigma}\left(\chi_{\Phi}\right)$ is denoted by the dashed (solid) curve. The PNJL calculations are done with three parameter sets of $\mathrm{A}, \mathrm{B}$ and $\mathrm{C}$ and these are distinguished by using different colors, green, blue and red, respectively.

Figure 6 shows the phase diagram of the chiral phase transition determined by $T_{\mathrm{c}}(\sigma)$. Green, blue and red curves are results of the PNJL calculations with sets $\mathrm{A}, \mathrm{B}$ and $\mathrm{C}$, respectively. In the entire region $0 \leq \theta \leq 2 \pi / 3$, the eight-quark interaction moves $T_{\mathrm{c}}(\sigma)$ down from the green dashed curve (set A) to the blue one (set B). However, the blue dashed curve still overshoots the lattice result (symbols) with $10 \%$ error near $\theta=\pi / 3$. The vector-type interaction makes the blue dashed curve go down to the red one (set $\mathrm{C}$ ) that is consistent with the lattice result [8]. Thus, the PNJL calculations with set $\mathrm{C}$ can reproduce the lattice result [4, 8] that $T_{\mathrm{c}}(\sigma)$ coincides with $T_{\mathrm{c}}(\Phi)$ within numerical errors in the entire region $0 \leq \theta \leq 2 \pi / 3$. 


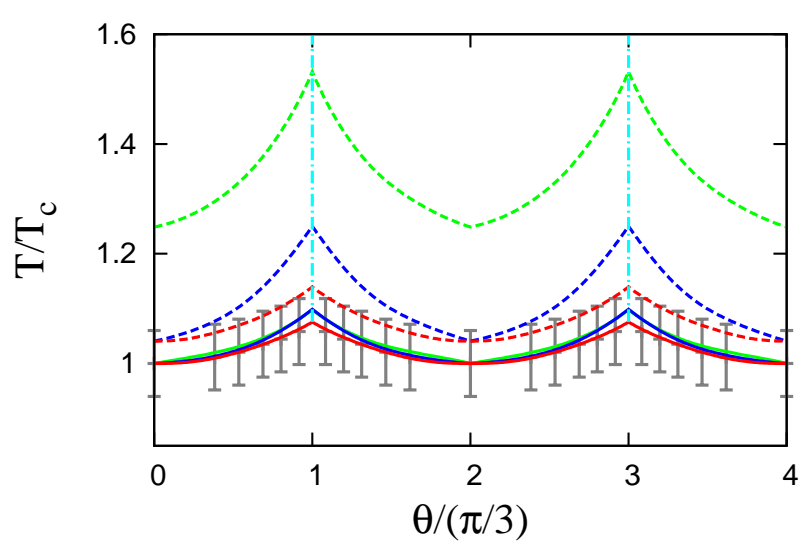

Fig. 6: Phase diagrams of the chiral phase transition in the imaginary chemical potential region calculated with three parameter sets are presented by dashed curves; green, blue and red ones are results of the PNJL calculations with set A, B and C, respectively. Lattice data [8] are shown with $10 \%$ error that $T_{\mathrm{c}}$ has [41]. The deconfinement phase transition curves (solid curves) are also shown for comparison.

Figure 7a) shows $\theta$ dependence of the phase $\phi$ of $\Phi$ for four cases of $T / T_{\mathrm{c}}=0.97,1.01,1.04$ and 1.10; each case is distinguished by using different colors. The PNJL results (curves) well simulate the lattice data [4, 8] (symbols). It is found from both the results that $\phi$ is continuous at $\theta=\pi / 3$ in the low- $T$ side $T \leq T_{\mathrm{RW}}=1.09 T_{\mathrm{c}}$, but it is discontinuous at $\theta=\pi / 3$ in the high- $T$ side $T>T_{\mathrm{RW}}$. Hence, the RW phase transition takes place at $T>T_{\mathrm{RW}}=1.09 T_{\mathrm{c}}$ and $\theta=\pi / 3$.

Figure 7 (b) shows $T$ dependence of $\phi$ for five cases of $\theta /(\pi / 3)=0,0.4,0.8,1.0$ and 1.2. The PNJL results (curves) well reproduce the lattice data [4, 8] (symbols). For $\theta<\pi / 3$ the phase $\phi$ tends to zero as $T$ increases, while for $\theta>\pi / 3$ it does to $-2 \pi / 3$ as $T$ increases. When $\theta=\pi / 3$, the RW phase transition takes place at $T>T_{\mathrm{RW}}=1.09 T_{\mathrm{c}}$ and then the phase $\phi$ is singular there, so that the pink line terminates at $T=T_{\mathrm{RW}}$. In the high- $T$ limit, the region (I) $-\pi / 3<\theta<\pi / 3$ has $\phi=0$ and the region (II) $\pi / 3<\theta<\pi$ does $\phi=-2 \pi / 3$. Thus, the region (II) is a $\mathbb{Z}_{3}$ image of the region (I), and the region (III) $\pi<\theta<5 \pi / 3$ is another $\mathbb{Z}_{3}$ image of the region (I).

\section{Thermal system with real chemical potential}

In this subsection, we predict the phase diagram in the real $\mu$ region by using the PNJL model. In Fig. 8, panels (a)-(c) represent results of the PNJL calculations with sets A, B and $\mathrm{C}$, respectively. Panel (c) is the most reliable result, since the PNJL result of set $C$ is consistent with the lattice result [4, 8] in the imaginary chemical potential region. Comparing the three panels, we find that the vector-type four-quark interaction and the scalar-type eight-quark interaction give sizable effects on the phase structure. In particular for the critical endpoint $\mathrm{E}$, the eight-quark interaction shifts point $\mathrm{E}$ to larger $T$ and smaller $\mu$, and the vector-type interaction moves it in
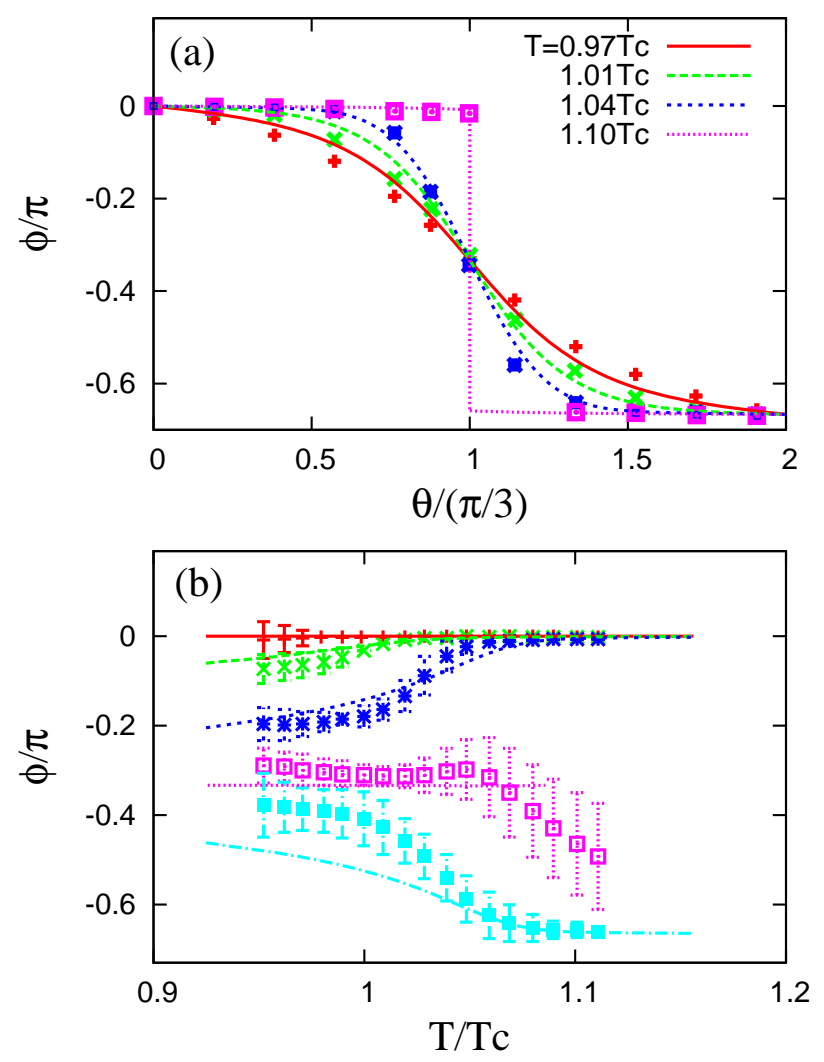

Fig. 7: Phase $\phi$ of the Polyakov loop as a function of (a) $\theta$ and (b) $T$. Lattice data [4, 8] are plotted by symbols. Curves represent results of PNJL calculations with set A. In panel (b), five cases (red, green, blue, pink and light blue) from top to bottom represent results of $\theta /(\pi / 3)=0,0.4,0.8,1.0$ and 1.2 , respectively. The pink line terminates at $T=T_{\mathrm{RW}}=1.09 T_{\mathrm{c}}$, since $\phi$ is singular at $T>T_{\mathrm{RW}}$ in the case of $\theta=\pi / 3$.

the opposite direction. On the red solid curve between point $\mathrm{E}$ and point $\mathrm{D}$ both the first-order chiral and deconfinement phase transitions take place simultaneously. The light-blue dot-dashed curve moving up from point I represents the RW phase transition of first order, and point $I$ is the critical endpoint. The green dashed curve between point $\mathrm{H}$ and point $\mathrm{E}$ means the crossover chiral phase transition and the blue solid curve between point $\mathrm{I}$ and point $\mathrm{E}$ does the crossover deconfinement phase transition. Point F (G) is a crossing point between the dashed (solid) curve and the $\mu=0$ line. Positions of points D-I are summarized in Table III. In panel (c), the pink dotted curve represents the lower bound of the location $\mu_{\mathrm{E}} / T_{\mathrm{E}}$ of the critical endpoint $\mathrm{E}$ that the LQCD analyses of Ref. [45] predict. The position of point $\mathrm{E}$ in the case of parameter set $\mathrm{C}$ is consistent with the results of the LQCD analyses.

\section{SUMMARY}

We have tested the reliability of the PNJL model, comparing the model result with lattice data in the imaginary chem- 


\begin{tabular}{ccccccc}
\hline set & D & E & F & G & H & I \\
\hline A & $(2.02,0.00)$ & $(1.84,0.72)$ & $(0.00,1.25)$ & $(0.00,1.00)$ & $(i \pi / 3 \times 1.53,1.53)$ & $(i \pi / 3 \times 1.09,1.09)$ \\
\hline B & $(1.68,0.00)$ & $(1.02,0.87)$ & $(0.00,1.05)$ & $(0.00,1.00)$ & $(i \pi / 3 \times 1.24,1.24)$ & $(i \pi / 3 \times 1.09,1.09)$ \\
\hline C & $(1.80,0.00)$ & $(1.51,0.72)$ & $(0.00,1.05)$ & $(0.00,1.00)$ & $(i \pi / 3 \times 1.13,1.13)$ & $(i \pi / 3 \times 1.07,1.07)$ \\
\hline
\end{tabular}

TABLE III: Positions of points D-I in $\mu$-T plane. The positions of these points are normalized as $\left(\mu / T_{\mathrm{c}}, T / T_{\mathrm{c}}\right)$ with $T_{\mathrm{c}}=173 \mathrm{MeV}$.
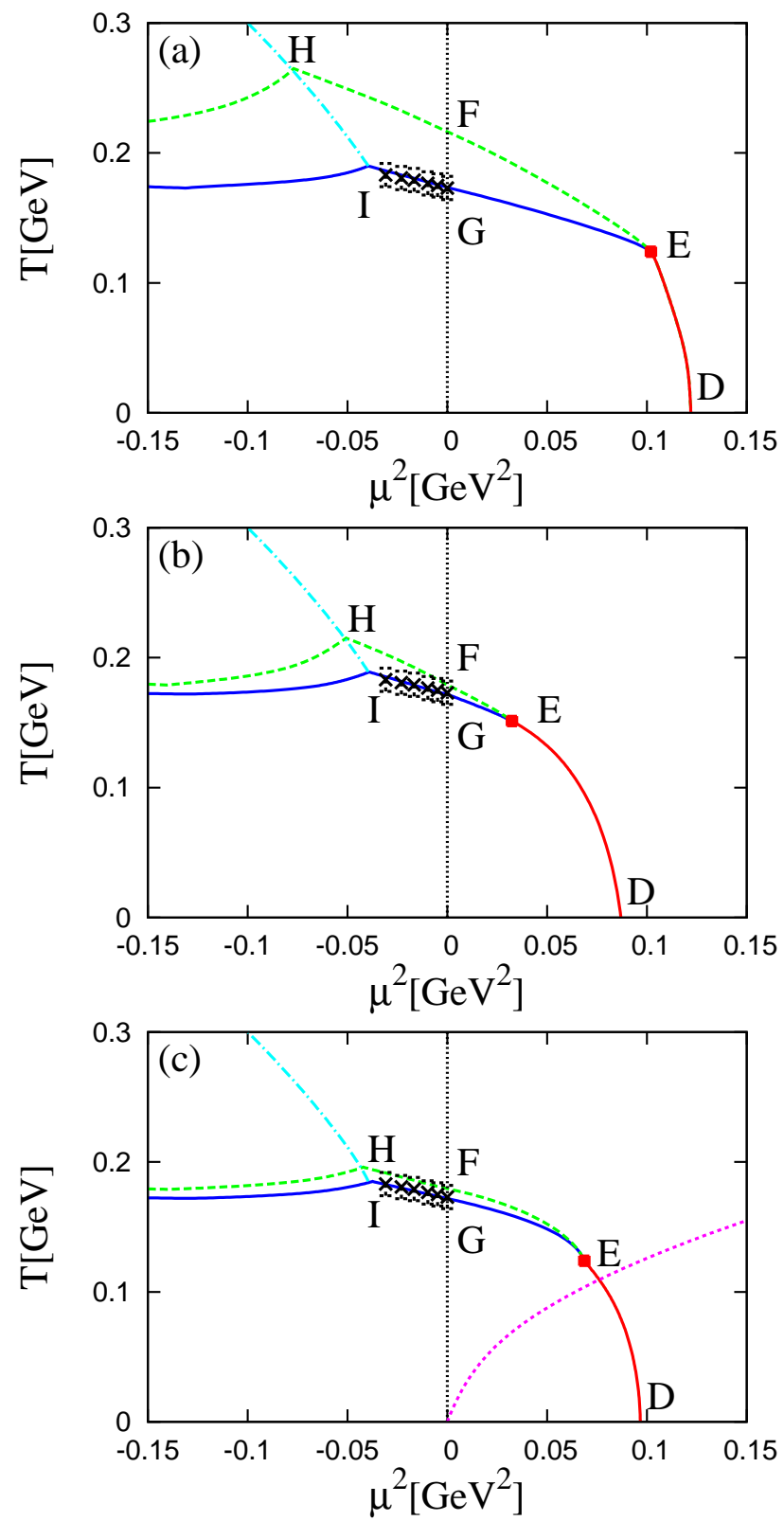

Fig. 8: Phase diagram in the real chemical potential region. (a), (b), and (c) are calculated with the parameter set A, B, and C, respectively. Cross symbols with error bars indicate the latticle data taken from Ref. [8]. Points D-I are explained in the text. ical potential $\left(\mu_{\mathrm{I}}=T \theta\right)$ region. In this test, the model parameters except $G_{\mathrm{v}}$ are adjusted so as to reproduce the measured pion mass and decay constant at $T=\mu=0$ and lattice data [40, 41, 42] at $T>0$ and $\mu=0$. In this step the eightquark interaction plays an important role to make $T_{\mathrm{c}}(\sigma)$ closer to $T_{\mathrm{c}}(\Phi)$ as discussed in our previous work [34]. With the aid of this, the PNJL calculation with the eight-quark interaction but without the vector-type interaction well reproduces the lattice data [4, 8] at finite $\theta$ on $\Phi$ and $T_{\mathrm{c}}(\Phi)$, but not on $T_{\mathrm{c}}(\sigma)$ particularly near $\theta=\pi / 3$ fully. The strength of $G_{\mathrm{v}}$ is then fitted so as to reproduce the data on $T_{\mathrm{c}}(\sigma)$ near $\theta=\pi / 3$. The primary result of the lattice simulations is that $T_{\mathrm{c}}(\Phi)$ coincides with $T_{\mathrm{c}}(\sigma)$, within numerical errors, in the entire region of $\theta$ [4, 8]. The PNJL model with the eight-quark and vectortype interactions can reproduce this property. Therefore, we can expect that the PNJL model with this parameter set is reliable also in the $\mu_{\mathrm{R}}$ region.

Finally, we quantitatively predict the phase diagram in the $\mu_{\mathrm{R}}$ region by using the PNJL model with the parameter set mentioned above. The critical endpoint does not disappear in virtue of the eight-quark interaction, even if the vector-type interaction is taken into account. This is the primary result of the present work. The lattice calculations at nonzero $\mu_{\mathrm{I}}$ have small lattice size $\left(8^{3} \times 4\right)[4,8]$. Therefore, it is highly expected that lattice simulations with larger size will be done in the $\mu_{\mathrm{I}}$ region.

\section{Acknowledgments}

The authors thank A. Nakamura for useful discussions and suggestions. K.K. and H.K. also thank M. Imachi, H. Yoneyama and M. Tachibana for useful discussions. This work has been supported in part by the Grants-in-Aid for Scientific Research (18540280) of Education, Science, Sports, and Culture of Japan. 
[1] J. B. Kogut and D. K. Sinclair Phys. Rev. D 77, 114503 (2008).

[2] Z. Fodor, and S. D. Katz, Phys. Lett. B 534, 87 (2002); J. High Energy Phys. 03, 014 (2002).

[3] C. R. Allton, S. Ejiri, S. J. Hands, O. Kaczmarek, F. Karsch, E. Laermann, Ch. Schmidt, and L. Scorzato, Phys. Rev. D 66, 074507 (2002); S. Ejiri, C. R. Allton, S. J. Hands, O. Kaczmarek, F. Karsch, E. Laermann, and C. Schmidt, Prog. Theor. Phys. Suppl. 153, 118 (2004).

[4] P. de Forcrand and O. Philipsen, Nucl. Phys. B642, 290 (2002);

[5] P. de Forcrand and O. Philipsen, Nucl. Phys. B673, 170 (2003).

[6] M. D'Elia and M. P. Lombardo, Phys. Rev. D 67, 014505 (2003); Phys. Rev. D 70, 074509 (2004); M. D’Elia, F. D. Renzo, and M. P. Lombardo, Phys. Rev. D 76, 114509 (2007);

[7] H. S. Chen and X. Q. Luo, Phys. Rev. D72, 034504 (2005); arXiv:hep-lat/0702025 (2007).

[8] L. K. Wu, X. Q. Luo, and H. S. Chen, Phys. Rev. D76, 034505 (2007).

[9] Y. Nambu and G. Jona-Lasinio, Phys. Rev. 122, 345 (1961); Phys. Rev. 124, 246 (1961).

[10] M. Asakawa and K. Yazaki, Nucl. Phys. A504, 668 (1989).

[11] J. Berges and K. Rajagopal, Nucl. Phys. B538, 215 (1999).

[12] O. Scavenius, Á. Mócsy, I. N. Mishustin, and D. H. Rischke, Phys. Rev. C 64, 045202 (2001).

[13] H. Fujii, Phys. Rev. D 67, 094018 (2003).

[14] M. Kitazawa, T. Koide, T. Kunihiro, and Y. Nemoto, Prog. Theor. Phys. 108, 929 (2002).

[15] A. A. Osipov, B. Hiller, and J. da Providência, Phys. Lett. B 634, 48 (2006); A. A. Osipov, B. Hiller, J. Moreira, and A. H. Blin, Eur. Phys. J. C 46, 225 (2006); A. A. Osipov, B. Hiller, J. Moreira, A. H. Blin, and J. da Providência, Phys. Lett. B 646, 91 (2007); A. A. Osipov, B. Hiller, J. Moreira, and A. H. Blin, Phys. Lett. B 659, 270 (2008); B. Hiller, A. A. Osipov, A. H. Blin, and J. da Providência, arXiv:hepph/0802.3193 (2008); B. Hiller, A. A. Osipov, J. Moreira, and A. H. Blin, arXiv:hep-ph/0809.2515 (2008); B. Hiller, J. Moreira, A. A. Osipov, and A. H. Blin, arXiv:hep-ph/0812.1532 (2008).

[16] K. Kashiwa, H. Kouno, T. Sakaguchi, M. Matsuzaki, and M. Yahiro, Phys. Lett. B 647, 446 (2007); K. Kashiwa, M. Matsuzaki, H. Kouno, and M. Yahiro, Phys. Lett. B 657, 143 (2007).

[17] T. Sakaguchi, M. Matsuzaki, H. Kouno, and M. Yahiro, Centr. Eur. J. Phys. 6, 116 (2008).

[18] P. N. Meisinger, and M. C. Ogilvie, Phys. Lett. B 379, 163 (1996).

[19] A. Dumitru, and R. D. Pisarski, Phys. Rev. D 66, 096003 (2002); A. Dumitru, Y. Hatta, J. Lenaghan, K. Orginos, and R. D. Pisarski, Phys. Rev. D 70, 034511 (2004); A. Dumitru, R. D. Pisarski, and D. Zschiesche, Phys. Rev. D 72, 065008 (2005).

[20] K. Fukushima, Phys. Lett. B 591, 277 (2004).

[21] K. Fukushima, Phys. Rev. D 77, 114028 (2008).
[22] S. K. Ghosh, T. K. Mukherjee, M. G. Mustafa, and R. Ray, Phys. Rev. D 73, 114007 (2006).

[23] E. Megías, E. R. Arriola, and L. L. Salcedo, Phys. Rev. D 74, 065005 (2006).

[24] C. Ratti, M. A. Thaler, and W. Weise, Phys. Rev. D 73, 014019 (2006); C. Ratti, S. Rößner, M. A. Thaler, and W. Weise, Eur. Phys. J. C 49, 213 (2007).

[25] S. Rößner, C. Ratti, and W. Weise, Phys. Rev. D 75, 034007 (2007).

[26] M. Ciminale, R. Gatto, N. D. Ippolito, G. Nardulli, and M. Ruggieri, Phys. Rev. D 77, 054023 (2008); M. Ciminale, G. Nardulli, M. Ruggieri, and R. Gatto, Phys. Lett. B 657, 64 (2007).

[27] H. Hansen, W. M. Alberico, A. Beraudo, A. Molinari, M. Nardi, and C. Ratti, Phys. Rev. D 75, 065004 (2007).

[28] C. Sasaki, B. Friman, and K. Redlich, Phys. Rev. D 75, 074013 (2007).

[29] B. -J. Schaefer, J. M. Pawlowski, and J. Wambach, Phys. Rev. D 76, 074023 (2007).

[30] P. Costa, C. A. de Sousa, M. C. Ruivo, and H. Hansen, arXiv:hep-ph/0801.3616 (2008); P. Costa, M. C. Ruivo, C. A. de Sousa, H. Hansen, and W. M. Alberico, arXiv:hep$\mathrm{ph} / 0807.2134$ (2008).

[31] K. Kashiwa, H. Kouno, M. Matsuzaki, and M. Yahiro, Phys. Lett. B 662, 26 (2008).

[32] W. J. Fu, Z. Zhang, and Y. X. Liu, Phys. Rev. D 77, 014006 (2008).

[33] H. Abuki, M. Ciminale, R. Gatto, G. Nardulli, and M. Ruggieri, Phys. Rev. D 77, 074018 (2008); H. Abuki, M. Ciminale, R. Gatto, N. D. Ippolito, G. Nardulli, and M. Ruggieri, Phys. Rev. D 78, 014002 (2008); H. Abuki, R. Anglani, R. Gatto, G. Nardulli, and G. Nardulli, Phys. Rev. D 78, 034034 (2008).

[34] Y. Sakai, K. Kashiwa, H. Kouno, and M. Yahiro, Phys. Rev. D 77, 051901(R) (2008); Phys. Rev. D 78, 036001 (2008).

[35] Y. Sakai, K. Kashiwa, H. Kouno, M. Matsuzaki, and M. Yahiro, Phys. Rev. D 78, 076007 (2008).

[36] A. Roberge and N. Weiss, Nucl. Phys. B275, 734 (1986).

[37] J. D. Walecka, Ann. Phys. 83, 491 (1974).

[38] T. Kashiwa and T. Sakaguchi, Phys. Rev. D 68, 065002 (2003).

[39] H. Kouno, T. Sakaguchi, K. Kashiwa, M. Hamada, H. Tokudome, M. Matsuzaki, and M. Yahiro, Soryushiron Kenkyu 112 C67 (arXiv:nucl-th/0509057) (2005).

[40] F. Karsch, Lect. notes Phys. 583, 209 (2002).

[41] F. Karsch, E. Laermann, and A. Peikert, Nucl. Phys. B 605, 579 (2002).

[42] M. Kaczmarek and F. Zantow, Phys. Rev. D 71, 114510 (2005);

[43] G. Boyd, J. Engels, F. Karsch, E. Laermann, C. Legeland, M. Lütgemeier, and B. Petersson, Nucl. Phys. B469, 419 (1996).

[44] O. Kaczmarek, F. Karsch, P. Petreczky, and F. Zantow, Phys. Lett. B 543, 41 (2002).

[45] S. Ejiri, arXiv:hep-lat/0706.3549 (2007); arXiv:heplat/0710.0653 (2007). 